\title{
The Effect of New Experimental System Design Related to the Plasma State on Achievement of Candidate Elementary Science Teachers
}

\author{
S. D. Korkmaz ${ }^{1, *}$, E.C. Aybek ${ }^{2}$, S. Pat ${ }^{3}$ \\ ${ }^{1}$ Department of Elementary Science Education, Eskisehir Osmangazi University, Meselik Campus, Turkey \\ ${ }^{2}$ Department of Educational Science, Eskisehir Osmangazi University, Meselik Campus, Turkey \\ ${ }^{3}$ Physics Department, Eskisehir Osmangazi University, Meselik Campus, Turkey
}

Copyright (C) 2015 by authors, all rights reserved. Authors agree that this article remains permanently open access under the terms of the Creative Commons Attribution License 4.0 International License

\begin{abstract}
The educational objectives related to the plasma state of matter, which comprises more than $90 \%$ of our universe, are located in the "properties of substances" unit in the 9th grade high school physics course curriculum. If there are physical and technical limitations while performing an experiment, the use of different techniques is recommended, such as demonstration experiments or simulations. Performing experiments related to the plasma state, which is the topic of this study, is very difficult in the classroom due to technical limitations. Thus, students cannot achieve the educational objectives related to the plasma state of matter. In addition, in Turkey, university curricula of elementary science education departments do not include lectures on plasma. The purpose of this study is to increase students' comprehension of the plasma state of matter and its properties. The newly designed experimental system and simulations are intended to answer the following questions: What is plasma? What are the general characteristics of plasma? How can plasma be created in the laboratory? For this purpose, a research group was established. The research group participants include :28 female and 4 male elementary science education teacher candidates from Eskisehir Osmangazi University. Data were collected by an achievement test which includes 10 multiple-choice and 10 essay-type questions (to determine the reasons behind students' answers) developed by the researchers. Essay questions were scored with a rubric. The achievement test was administered before and after the experiment, and mean scores were compared by an independent samples t-test. In addition, the students' answers to the essay-type questions were compared by their rubric scores. The results of the independent samples t-test indicated that the students' mean post-test score was significantly higher than their mean pre-test score. In addition, rubric scores showed that students answered multiple-choice questions based on a higher level of understanding of plasma.
\end{abstract}

Keywords Teaching of Plasma State, Science Education, Candidate Elementary Science Teachers

\section{Introduction}

There are four matters of state in the universe: Solid, liquid, gas and plasma states. In case of a solid body, molecules constituting the solid body move less. When the average energy of molecules is increased by any method (for example, with heat), the body is transformed first to liquid, then to gas. Electrons in the gas phase move quickly. If one continues to apply heat upon the gas state, ionization might start. In an ionization state, at least one electron leaves an atom or molecule. Plasma obtains a high electrical conductivity due to free electrical charge and is affected easily by electromagnetic fields. Ionization occurs repeatedly in a sufficiently warmed gas, and free electron and ion clouds begin to form. However, some atoms are in an excited-state condition and continue to remain electrically neutral, and a photon is emitted to release excess energy, making the atom basic. This medium, in which electrons, ionized atoms and molecules, and neutral atoms and molecules exist at an excited level, and photons are released, and all particles interact with each other permanently, is called "plasma." The total charge of plasma is zero, meaning that collectively, it is a neutral state of matter $[1,2]$

The plasma state was first defined by Irving Langmuir in 1929 as ionized constituents of more than $95 \%$ of the universe. Examples of plasmas in our solar system include the sun, solar winds, supernovas, plasmas in nebular universe, polar lights (Aurora), lightning, flash, fire, ionosphere layer of the earth, and the Van Allen belt. Furthermore, examples of plasmas generated in vitro include neon advertising lights submitted by state-of-the-art technology, xenon headlights of cars, sodium vapor lamps, and more [1-4]

The reason why we encounter the plasma state of matter quite frequently is that this state of matter has quite different properties than its other states. The two most important plasma parameters are charged particle density (number of particles $/ \mathrm{m}^{3}$ ) and temperature $(\mathrm{K})$, and plasmas may be classified depending on these parameters. In Figure 1, a 
temperature variation by particle density is shown schematically $[1,2,5]$

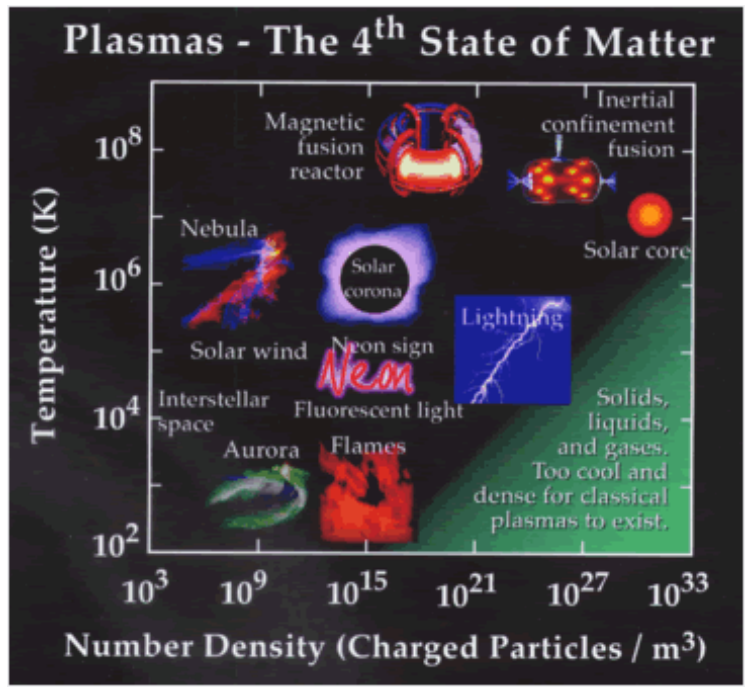

Figure 1. Plasma temperature vs. number density of particles (Contemporary Physics Education Project, http://www.cpepweb.org/).

The concept of plasma plays a basic role in current research and development activities. However, it is difficult for science and engineering undergraduates to understand the basic properties of plasma [6]. The undergraduates must be able to learn and apply electromagnetic theory, dynamics, thermodynamics, fluid mechanics, statistical mechanics and chemical equilibrium associated with the plasma state of matter to understand plasma and its properties. Although this is possible for science and engineering undergraduates in Turkey who reach a master degree level, understanding plasma is more difficult for undergraduates in the department of science teaching in education degree programs. Undergraduate students in the science teaching program learn the subjects as specified above in physics courses at a basic level during the first two years of the 4-year program. However, there is no course in which the plasma state is described in master degree programs in the science teaching department [7].

\section{Aim of the Study}

The aim of this study is to enable the candidate science teachers to better understand the plasma state of matter, which comprises the majority of the universe and is used today to manufacture and generate novel materials and power, and its properties. An experimental demonstration was designed for the undergraduates, and this study examines whether there is a significant change in the candidate teachers' knowledge of plasma before and after this experimental demonstration.

\section{Model of Research}

This research is a quasi-experimental study that aims at examining any change in knowledge of the candidate teachers on plasma before and after application of the experimental system.

\section{Study group}

The study group consisted of 32 candidate teachers, including 28 women and 4 men, who were studying in the $3^{\text {rd }}$ year of the Elementary Science Teaching Department of Educational Faculty of the Eskisehir Osmangazi University.

\section{Data acquisition tools}

Plasma Achievement Test (PAT) (Appendix-A): An achievement test consisted of 10 multiple-choice questions and 10 open-ended questions was developed. In the open-ended questions, the candidate teachers were expected to justify any answers given to the questions. Questions on the test were developed to reveal the candidate teachers' knowledge of plasma and how they use this knowledge. In developing the test questions, it was assumed that the candidate teachers had courses related to plasma state in the $1^{\text {st }}$ and $2^{\text {nd }}$ years of education school. Care was taken to include questions that assessed any acquisition of knowledge that the candidate teachers might have on plasma to ensure validation of the scope of the test. We consulted with two measurement and evaluation specialists and two physics teachers for their opinion on achievement test. One of two measurement and evaluation specialists is Ph.D. student at Division of Measurement and Evaluation in Education of the Ankara University Graduate of Educational Sciences, and the other one has Ph.D. degree at Educational Administration, Supervision, Planning and Economy and has taught measurement and evaluation courses to candidate elementary teachers for three years. Both physics teachers work at Eskişehir Fatih Anatolian High School and one of them has taught $9^{\text {th }}, 10^{\text {th }}, 11^{\text {th }}$ and $12^{\text {th }}$ grade physics courses of secondary school curriculum for 18 years and the other one has taught for 17 years. Additionally, one physicist who has taught dynamics, modern physics, and electromagnetic theory courses of undergraduate degree programs of physics department since 1994 and whose published papers are related to thin film deposition by using plasma source and one science teaching specialist who has Ph.D. degree at Elementary Science Teaching and works at Elementary Science Education Department of ESOGÜ were consulted for their opinions. A scaled scoring key was used that was prepared by asking two physicist who have Ph.D. degree at Plasma Physics and one measurement evaluation specialist who is Ph.D. student at Division of Measurement and Evaluation in Education for their opinions on scoring of the open-ended questions of the academic achievement test. Accordingly, both the multiple-choice questions and the open-ended questions were scored to total 100. In scoring, each item was given equal weight.

\section{Procedure}

A new experimental system was designed as an 
experimental demonstration in accordance with the goal of the study. The experimental system shown in Figure 2 consists of seven discharge tubes and a plasma stick. Discharge tubes filled with different gases (Ar, Neon, etc.) at different pressures. Electrodeless discharge generation was ensured by using plasma stick isolated by a glass tube with a high voltage $(3-4 \mathrm{kV})$ and a high frequency $(10 \mathrm{kHz})$. Because gas pressures and gas types are different in the tubes, different color plasmas occur. It can be explain to the students why plasmas occur in different colors.

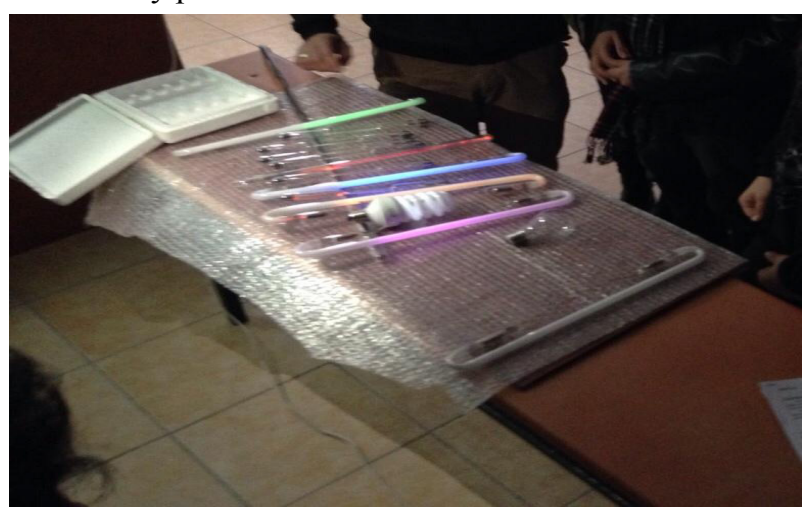

Figure 2. Experimental set-up

As a pre-test, the PAT was administered to the candidate teachers one week prior to the experimental demonstration. During the experimental demonstration, the Neon Lights \& Other Discharge Lamps simulation was used [8], and the PAT was applied again as a post-test.

\section{Data analysis}

To prepare data for analysis, the primary procedure was to assign each multiple-choice item a score of 1 for each correct answer and 0 for each incorrect answer. Then, the total number of correct answers for each candidate teacher was calculated for both the pre-test and post-test. For easier interpretation, scores were converted to a 100 -score system. To do this, scores were multiplied by 10 in the conversion because there were 10 multiple-choice questions in the test.

In scoring answers given by the undergraduates to the multiple-choice questions, each item was scored out of 100 scores by means of a scaled scoring key.

Answers given by the candidate teachers to the multiple-choice and open-ended questions in the test were examined by a frequency analysis, differences between pre-test and post-test scores were examined, and the score differences were analyzed to see if they met the normality hypothesis. To that end, the Shapiro-Wilk normality test was applied, and it was determined that the distribution of score differences doesn't differ excessively from a normal distribution for both multiple-choice questions and for justifications $(p>.05)$. Accordingly, the t-test was used as a parametrical test for correlated samples in average comparisons.

\section{Findings}

Pre-test and post-test score averages of the candidate science teachers were compared by considering their answers to the multiple-choice questions and their justifications for these answers.

Accordingly, pre-test and post-test comparisons of average scores gained by the candidate teachers in the multiple-choice questions are given in Table 1.

Table 1. t-Test Results of Correlated Samples for Multiple-Choice Questions.

\begin{tabular}{ccccccc}
\hline Test & $\mathrm{N}$ & $\overline{\mathrm{X}}$ & $\mathrm{S}$ & $\mathrm{df}$ & $\mathrm{T}$ & $\mathrm{p}$ \\
\hline Pre-test & 32 & 57.19 & 14.64 & 31 & -6.271 & .000 \\
Post-test & 32 & 75.31 & 12.95 & & & \\
\hline
\end{tabular}

As shown in Table 1, there was a significant increase in average score gained by the candidate teachers in the multiple-choice questions from the pre-test to the post-test $\left(\mathrm{t}_{31}=-6.271 ; \mathrm{p}<.05\right)$. To determine the magnitude of the effect of this significant difference, Cohen's d coefficient was calculated and determined to be $d=1.10$. This value notes a large difference between the pre-test and post-test scores.

A t-test was used to determine whether there was a significant difference in average scores of the candidate teachers in the open-ended questions between the pre-test and the post-test; these results are shown in Table 2.

Table 2 . t-Test Results of Correlated Samples for Justifications

\begin{tabular}{ccccccc}
\hline Test & $\mathrm{N}$ & $\overline{\mathrm{X}}$ & $\mathrm{S}$ & $\mathrm{df}$ & $\mathrm{T}$ & $\mathrm{p}$ \\
\hline Pre-test & 32 & 34.84 & 9.94 & 31 & -4.454 & .000 \\
Post-test & 32 & 41.84 & 7.13 & & & \\
\hline
\end{tabular}

As shown in Table 2, there was a significant increase in averages score gained by the candidate teachers in the open-ended questions from the pre-test to the post-test $\left(\mathrm{t}_{31}=\right.$ $-4.454, \mathrm{p}<.05)$. To determine the magnitude of the effect magnitude of this significant difference, Cohen's $d$ coefficient was calculated and determined to be $d=.79$. This value implies a large difference between the pre-test and post-test scores.

Descriptive statistics showing the questions for which the candidate teachers had the most and least success according to post-test are given in Table 3 .

Table 3 shows that the candidate teachers were most insufficient in answering multiple-choice questions 2 and 10 compared to other questions. 
Table 3. Number of correct and incorrect answers given to the multiple-choice questions

\begin{tabular}{ccc}
\hline Question & Correct & Wrong \\
\hline 1 & 32 & 0 \\
2 & 17 & 15 \\
3 & 30 & 2 \\
4 & 21 & 11 \\
5 & 21 & 11 \\
6 & 31 & 1 \\
7 & 30 & 2 \\
8 & 23 & 9 \\
9 & 23 & 9 \\
10 & 13 & 19 \\
\hline
\end{tabular}

Table 4 shows the number of undergraduates who gained five scores or less according to the scaled scoring key for the open-ended questions.

Table 4. Number of Individuals Who Gained Five Scores or Less from Answers Given to the Open-Ended Questions

\begin{tabular}{cc}
\hline Question & Five Scores and less \\
\hline 1 & 0 \\
2 & 31 \\
3 & 32 \\
4 & 31 \\
5 & 29 \\
6 & 29 \\
7 & 15 \\
8 & 15 \\
9 & 29 \\
10 & 27 \\
\hline
\end{tabular}

The third, sixth and ninth questions were remarkable; for these questions, although the candidate teachers gave correct answers to the multiple-choice type questions, their given answers were not justified according to the corresponding open-ended questions.

\section{Discussion and Conclusion}

This study showed that the average post-test scores of the candidate teachers were significantly higher than the averages pre-test scores. This increase in post-test scores was observed in both multiple-choice questions and open-ended questions. In this study, it might be interpreted that the candidate teachers were better able to justify their answers after the experimental demonstration.

Examination of the answers given by the teacher candidates to the multiple-choice and essay-type questions uncovered that they gave the correct answers but without an apparent justification to the third, sixth and ninth questions. Due to the nature of the multiple-choice questions, the question is presented with options, and the individual and is expected to locate and mark the correct answer from the available options. If knowledge has been constructed, the individual is able to give correct answers to the essay-type questions. Therefore, for the third, sixth and ninth questions, the teacher candidates selected the correct answer from the options given in the multiple-choice questions but were unable to explain their responses. When these three questions are examined, it is noted that they pertain to the basic properties of plasma and natural and artificial plasma samples. This result shows that the knowledge related to the basic properties of plasma had not been constructed by the teacher candidates.

The findings of this research can be interpreted with the following suggestions:

Teaching of plasma must be supported by experiments and simulations.

In future research, students' responses to essay-type questions should be more thoroughly investigated with a qualitative research design.

The students' scores on multiple-choice and essay-type questions were significantly increased by the experimental setup developed by this research. However, their responses to certain items show that students could not justify their correct answers. When developing the plasma experiment, its setup can be modified after the findings of qualitative research.

\section{Acknowledgements}

This study was supported by the Eskisehir Osmangazi University Scientific Research Projects Committee (Project No: 201421027). The authors gratefully acknowledge the financial support from the organization. 


\section{Appendix}

\section{Plasma Achievement Test (PAT)}

Dear students:

This test consists of 10 questions. Each question has two steps. A multiple-choice question constitutes the first step of the questions, and another question, which requires describing reasons for the answer given to that question, constitutes the second step.

Any clear and understandable answers that you give to the questions will give us critical hints on new educational and teaching techniques. The answers that you will give will have no score value and will be kept confidential.

We thank you for your participation and wish you success.

In how many states of matter exist in the universe?
A) 3
B) 4
C) 5
D) 6
E) 7

I. Gases are not affected by magnetic and electrical fields, but plasmas are affected by magnetic and electrical fields.

II. Because the distance between molecules of gases is large, gases do not conduct heat well, but plasmas do conduct heat well.

III. Gases and plasmas do not conduct electricity.

Which of the above phrases is/are true?
A) Only I
B) Only II
C) Only III
D) I and II
E) I and III

Type your reason for $\mathrm{S}-2$ briefly.

I. It consists of ionised and excited atoms.

II. Because it has an equal number of positive charges (ion charges) and negative charges (electrons), it is neutral electrically.

III. It consists of an ionised gas.

Which of the above phrases about the plasma state of matter is/are true?
A) Only I
B) Only II
C) Only III
D) I and II
E) I, II and III

Type your reason for $S-3$ briefly.

An atom:

I. Can be heated and excited.

II. Can be excited by electromagnetic waves (photons).

III. Can be excited by electron bombardment.

Which one/s of the above consideration is/are true?
A) Only I
B) Only II
C) Only III
D) II and III
E) I, II and III

Type your reason for $\mathrm{S}-4$ briefly.

When a high voltage is applied to both ends of a glass tube containing a low pressure gas, the tube emits light (e.g., neon lights in billboards).

\section{What is/are physical reason/s for this radiation?}

A) Due to the applied high voltage, atoms in the tube combine and form molecules. Meanwhile, the liberated energy is seen as a light.

B) As a result of collusions, the order of electron of atoms in the tube changes, and atoms irradiate while they restore to their original state.

C) Due to a potential difference applied to the ends of the tube, a current passes through the tube. This current causes irradiation just like in wired bulbs.

D) Electrons moving inside the tube irradiate.

E) Positive ions moving inside the tube irradiate.

Type your reason for $\mathrm{S}-5$ briefly.

Which of the following definitions of plasma is incorrect?

A) It is a stimulated state of gas.

B) It is a warmed state of gas.

C) It is a high-energy state consisting of ions, neutral atoms, free electrons and photons.

D) It is a state at a vaporisation point.

E) It is a state at a sublimation point. 
Type your reason for $S-6$ briefly.

Which one of the following items is not an example of plasma?
A) Lightning
B) Incandescent lamp
C) Flash
D) Fluorescent lamps
E) Matter in the Sun

Type your reason for $S-7$ briefly.

In which of the mechanisms shown in the right figure can the lamp be expected to emit light?
A) Only I
B) Only II
C) Only III
D) I and II
E) II and III

I

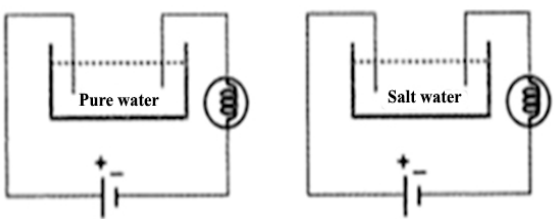

III
II

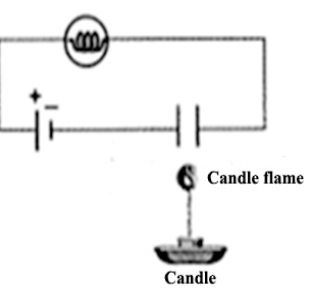

Type your reason for $\mathrm{S}-\mathbf{8}$ briefly.

Which of the following phrases is/are true?

I. Fluorescent and neon lamps are examples of plasma produced in vitro.

II. Sun wind is an example of plasma in space.

III. Auroras are examples of plasma occurring on the Earth.
A) I and II
B) II and III
C) Only III
D) I and III
E) I,II and III

\section{Type your reason for $\mathrm{S}-9$ briefly.}

I. Energy production with plasma is cleaner and causes less damage to the environment.

II. Plasma technology is used in industry and in cleaning from bacteria and other microorganisms.

III. Plasma in stars is called "hot plasma."

Which one/s of the above consideration is/are true?
A) I and II
B) I and III
C) II and III
D) I, II and III
E) Only III

Type your reason for $S-10$ briefly.

ANSWER KEY TO THE ACADEMIC ACHIEVMENT TEST
1. $\mathrm{B}$
2. D
3. $\mathrm{E}$
4. $\mathrm{E}$
5. B
6. $\mathrm{C}$
7. $B$
8. E
9. $\mathrm{E}$
10. D 


\section{REFERENCES}

[1] Alfred Grill, (1993), Cold Plasma in Materials Fabrication From Fundamentals to Applications, IEEE Press, New york,

[2] S.Elizer, Y.Eliezer( 2001), The Fourth State of Matter An Introduction to Plasma Science, IOP publishing,

[3] Contemporary Physics Education Project, http://www.cpepweb.org/ Accessed on 26.09.2014

[4] Ian Tresman, The Plasma Universe Wikipedia-like Encyclopedia, http://www.plasma-universe.com/Plasma-Universe.com, Accessed on 26.09.2014

[5] ESOGU Plasma science and technologies lab home page, http://esoguplazma.ogu.edu.tr, Accessed on 26.09.2014

[6] Porter Wear Johnson, Lectures in Plasma Physics, Chicago, Illinois file://D:/E\%C4\%9Fitim\%20yay\%C4\%B1n/BAP\%20Proje/ plasmaweb.pdf Accessed on 29.12.2014

[7] T.C. Legislation of the Higher Education Institute, Faculty of education Degree programs of Teacher education, Page 53, https://www.yok.gov.tr/documents/10279/30217/E\%C4\%9E $\% \mathrm{C} 4 \% \mathrm{~B} 0 \mathrm{~T} \% \mathrm{C} 4 \% \mathrm{~B} 0 \mathrm{M}+\mathrm{FAK} \% \mathrm{C} 3 \% 9 \mathrm{CLTES} \% \mathrm{C} 4 \% \mathrm{~B} 0 \% 20$ $\% \mathrm{C} 3 \% 96 \% \mathrm{C} 4 \% 9 \mathrm{ERMEN}+\mathrm{YET} \% \mathrm{C} 4 \% \mathrm{~B} 0 \% \mathrm{C} 5 \% 9 \mathrm{ET} \% \mathrm{C} 4$ $\%$ B0RME+L $\%$ C4\%B0SANS+PROGRAMLARI.pdf/054dfc 9e-a753-42e6-a8ad-674180d6e382 Accessed on 29.12.2014

[8] PHET interactive simulations, https://phet.colorado.edu/tr/simulations/category/physicsAcc essed on 29.12.2014 